obtenir le rendement optimum, chaque Salmoniculteur fera le recensement de ses possibilités d'approvisionnement en produits d'origine animale et végétale. Pour composer son mélange il n'oubliera pas que les vitamines sont indispensables et qu'elles sont apportées : - par la viande fraîche, saine, non stérilisée ; - par le Poisson frais non congelé, - ct par certains produits d'origine végétale, levure, drêche, etc. Il ajoutera à l'aliment porteur de vitamines le complément d'origine végétale ou le produit commercial qu'il jugera convenable. Il lui appartiendra, par des essais suivis et répétés, de rechercher le mélange " au goût " de la Truite, ainsi que le dosage et le mode de trituration pour qu'il soit pris facilement et assimilé commodément.

Il semble que, pour une mise au point rapide, la collaboration du Laboratoire et du Salmoniculteur doive être féconde, par les renseignements que les analyses biochimiques ef l'expérimentation donneront sur les progrès de l'élevage.

La question de l'alimentation étant d'une importance capitale pour la Salmoniculture, les critiques, remarques, suggestions, résultats d'expérience, etc., que les Pisciculteurs voudraient bien exprimer seront accueillis avec reconnaissance, discutés et publiés dans l'intérêt général.

\title{
L'EXPLOITATION DES ÉTANGS
}

\author{
Par M. Primlibert PANNETIER \\ Secrétaire général de l'Union nationale des Pêcheurs aux filets.
}

(Suite) (I)

Choix et production des alevins.

Du choix des alevins dépend, pour une grande part, la récolte de l'annéc suivante, aussi convient-il d'insister sur cette question.

Je commencerai par les alevins de Carpes d'un été (feuilles ou nourrains, suivant la région où l'on se place), destinés à faire, l'année suivante, des carpailles ou pannots. A priori, le choix de ces alevins, que ce soit en sijectionnés ou en communs, n'a pas une très grande importance quant ¿ la grosseur, étant donné que l'on a vu de très petites feuilles (désignées sous le nom d'amandes, dans certaines régions) faire souvent, au bout (i'ume année, de très beaux pannots.

Néanmoins, il est toujours bon, dans une pèche où il y a abondance et cmbarras du choix, de choisir les plus beaux sujets, qui sont toujours plus robustes, pour éviter des pertes parfoị très élevées au cours du transport. Nais, en dehors de cette question de résistance, les petites - les imandes -, ne sont pas à craindre, étant donné que ce n'est pas le mauque de croissance qui leur a fait défaut, mais seulement l'âge.

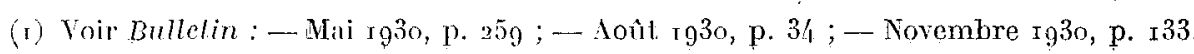


Il arrive assez souvent que tous les géniteurs, ou seulement une partie, en raison notamment de la température de l'eau, ne posent qu'en Juillet cu Août, au lieu de Mai. Il en résulte que les alevins de ces poses, lors de ia pêche en Novembre, n'ont, en réalité, que trois ou quatre mois d'existence et que, même pêchés en Février ou Mars, ils n'ont que sept ou huit mois de vie. Donc, rien de plus naturel que de trouver dans la mềme pêche des alevins présentant des différences du simple ạu double, ou, même du simple au triple, sans que les uns et les autres soient défectueux pour cela.

Pour éviter, dans la mesure du possible, des pertes en empoissonnant avec des alevins de trois ou quatre mois, un surcroît de précaution s'impose, surtout s'il y a lieu de procéder à un long transport pour l'empoissonnement.

En ce qui concerne les alevins de Carpes de deux années (carpailles ou pannots), la question se pose d'une toute autre façon. Qu'il s'agisse d'alevins d'origine sélectionnée ou non, étant donné que les plus petits sont' assurément les moins voraces, ceux qui végéteront au cours de l'année ou des années suivantes, un choix judicicux s'impose lorsqu'jl y a possibilité. C'est, d'ailleurs, en éliminant ces sujets faibles et médiocres, que: l'on arrive à faire de la sélection et à régénéver la race.

En procédant ainsi lors d'un empoissonnement, et en ne surchargeant pas outre mesure en alevin quel qu'il soit, il $y$ bien des chances que l'on pêche l'année suivante de très belles Carpes ayant peu de différence comme poids. Si au contraire, on empoissonne avec n'importe quels alevins, on récoltera toujours des Carpes, bàtardes, suivant l'expression vulgaire, c'est-à-dire pesant depuis 200 grammes jusqu'à a ou 3 livres.

Ce point établi, passons à un autre.

J'ai entendu souvent des propriétaires d'étangs faire, à propos des pannots pesant en moyenne 400, 500, même 600 grammes pièce, cette réflexion : "A quoi bon emporssonner avec de si beaux sujets, coûtant très cher, alors qu'à leur pêche, la plupart du temps, les Carpes ne seront pas plus grosses que si les pannots avaient été de grosseur moyenne?"

Leur raisonnement est parfois juste : il arrive qu'avec de très beaux alevins de deux ans, certains pisciculteurs, ou certains étangs, ne font que des Carpes ordinaires ou peu au-dessus de la moyenne. Dans ceci, il n'y a rien de surprenant ; je vais, dans la mesure de mes moyens, en donner. une explication.

Même en admettant que nous ayons à faire à des poissons d'origine sélectionnée, pour récolter des alevins de Carpes de deux années, pesant au-dessus de 400 grammes pièce, il faut que l'étang d'où ils proviennent n'ait pas été chargé, que la nourriture y ait śté surabondante et que, d'autre part, l'eau se soit maintenue à une température assez élevée.

Or, pour que ces alevins continuent ì croitre dans les mêmes proportjons que précédemment, il faudrail: 
$I^{\circ}$ Qu'ils fussent déversés sur un fond équivalent, comme qualité, à celui d'où ils proviennent ;

$2^{\circ}$ Que l'empoissonnement fût fait en tenant compte, non seulement du nombre, mais surtout du poids.

Malheureusement, le contraire se produit le plus souvent. Des pannots dc toute beauté, habitués à la bome vie, provenant d'un très bon fond, bien situé, sont déversés dans un ćlang placé au milieu des bois, recevant des eaux froides et dépourvies de nourriture. Rien de plus naturel que ces poissons se ressentent de cette différence de conditions d'existence et ne profitent pas comme précédemment ! Sans aucun doute, des pannols, même plus petits, élevés, acclimatés dans un fond identique auraient donné d'aussi bons résultats.

D'autre part, même en admettant que le déversement ait lieu dans un fond équivalent en qualité à celui où les pannots ont été pêchés, beaucoup de propriétaires, sans tenir compte de la grosseur des alevins et de labondance de la nourriture, qui, de ce fait, leur est nécessaire pour continuer leur rapide croissance, empoissonnent en nombre, dans les mêmes proportions qu'avec des alevins de poids moven. Certains même chargent encore davantage. Dès lors, rien d'étonnant que ces alevins de deux années (carpailles ou pannots) ne continuent pas leur vive progression de leur deuxième année, au cours de la troisième et même de la quatrième, si l'étang ne se pêche que tous les deux ans.

En résumé, quand il s'agit d'empoissonnage de Carpes de deux étés, d'un poids bien au-dessus de la moyenne, il faut abandonner le principe d empoissonnement à la tête ou au cent, pour pratiquer, comme je le disais dans un de mes précédents articles, celui au poids, de i 5 à 20 kilogrammes à l'hectare.

Ce procédé permettra de réaliser un gros bénéfice sur l'empoissonnage, es de récolter de belles Carpes, ayant une valeur marchande incontestabiement supérieure, sans que le poids total de la pêche ait à en souffrir.

En ce qui concerne les Tanches, le choix des alevins, sans avoir la même importance que pour les Carpes, influe néanmoins, pour une grande part, sur la qualité et la quantité de la marchandise à la récolte. J'ai vu de gros propriétaires faisant valoir jusqu'à cent hectares d'étangs en bon état, négliger complètement leur empoissonnement de Tanches ; remettre indéfiniment, à chaque pêche, des tanchons déjà pêchés plusieurs fois, n'ayant que l'échine et ne pesant guère plus à leur quatrième année qu'à leur deuxième. En procédant ainsi, on ne trouve pour ainsi dire pas de Tanches marchandes aux pêches suivantes, mais uniquement des lanchons dégénérés.

Il y a lieu de se débarrasser, à n'importe quel prix, de toutes les Tanches n'ayant pas atteint la taille marchande à la troisième année. Fnsuite, si l'empoissonnement de l'étang en Carpe est normal, c'est-àdire de 15 à 20 kilos à l'hectare, on ne chargera pas en Tanches (ro kilos à l'hectare au maximum). 
En procédant ainsi, l'année suivante, lors de la pêche, toutes les Tanches, marchandes ou non, doivent ètre livrées à la consommation.

Ceci dit, peut-on reconnaître les alevins de Tanche de un et de deux étés (éguillons et tanchons) pour assurer leur production Il est assez difficile de faire un choix judicieux entre des alevins d'un el de deux étés et, d'autre part, de différencier ceux-ci des Tanches de trois étés, lorsque celles-ci n'ont pas pas profité normalement.

Il y a donc lieu d'en faire l'élevage séparément, pour qu'aucun doute ise puisse subsister, quant à leur àge à la pêche, et permettre de régénérer les empoissonnages.

Pour cela, il n'y a qu'à peupler les étangs d'alevinage, de la façon suivante :

Carpes de pose et Tanches de pose seulement.

Ou encore :

Carpes de pose et alevins d'un été (feuilles).

Tanches de pose.

L'année suivante, il y aura lieu, pour éviter toute confusion, d'empoissonner de cette façon :

Carpes de pose avec ou sans alevins d'un été (feuilles) suivant le procédé adopté pour la culture des alevins de Carpes, alevins de Tanches d'un èié (éguillons), pour obtenir des tanchons l'annéc suivante.

De cette façon le choix des alevins se fait automatiquement, aucune confusion n'étant possible. En ce qui concerne les Tanches, il y aura dans l'étang: - la première année : Tanches de pose et éguillons ; - la deuxième année : Tanchons (alevins de deux étés) seulement.

Ceci, bien entendu, à condition que l'étang d'alevinage sorte de l'assec, cu ait été pêché à fond, de façon qu'il n'y reste absolument rien, comme empoissonnage, des pêches précédentes.

Comme conclusion, en admettant que l'on se soit conformé strictement aux indications précédemment données, quant au choix des géniteurs, les Tanches issues de ccux-ci, quel que soit leur poids, ayant réellement deux années d'existence, feront sans aucun doute, s'il n'y a pas surcharge, des sujets de 250 à 500 grammes au cours de la troisième année, sur des fonds en bon état.

D'autre part, on obtiendra un rendement qui ne sera pas inférieur à fo kilogrammes à l'Hectare et peut aller jusqu'à 60 si les circonstances sont favorables.

En ce qui concerne les alevins de "blane ", le choix n'a pas beaucoup d'importance, car les petits se vendent, dans la région lyonnaise, mieux que les gros. Néanmoins il faut, autant que possible, mettre dans un étang, alevins de $\mathrm{I}$ et 2 étés. Les premiers, pour alimenter les filatons et brochetons, les deuxièmes pour nourrir les Prochets et, ensuite, assurer ''empoissonnement de l'année suivante.

Ces Cyprinides sont tellement prolifiques qu'il n'y a pas lieu de s'inquiéter pour les alevins. Inutile de faire de l'cmpoissonnage dans des ètangs spéciaux. Dès leur deuxième année, dans les étangs de pêchc, réglée, 
ils posent et il n'est pas rare de voir un seul étang fournir de quoi empoissonner tous ceux d'une région. Aussi, c'est par milliers de kilogrammes que des alevins de I et de 2 étés de ces Cyprins, sont livrés à la consommation dans la région des Dombes ou les procédés de pêche permettent une exploitation judicieuse de ces espèces et la conservation de leurs alevins, si petits soient-ils.

Successivement, viennent d'être traitées les qucstions de la production of du choix des alevins de Carpe, de Tanche et de blanc, il ne me reste donc qu'à parler du Brochet, Poisson vorace par excellence, mais à beaucoup de points de vue, indispensable dans les ètangs.

Vltérieurement, à propos des proportions d'alevins à employer pour l'empoissonnement des étangs de pêche réglée, seront données les raisons pour lesquelles est jugée utile la présence, en nombre limité, de Brochets dans les étangs de poissons de vente comestjble. Bornons-nous, pour l'instant, à exposer comment doit ètre fail le choix des alevins pour l'emroissonnage.

Lc Brochet étant très prolifique el de croissance très rapide, il n'est' pas nécessaire d'en fairc l'élevage indépendamment des autres espèces, pour assurer l'approvisionnement des étangs de pêche réglée où -ils sont utiles. Ceux-ci ne réclament aucun soin particulier et aucun choix n'est a a fairc pour les Brochets de pose. Tous les brochetons, même de moins de roo grammes, sont susceptibles de poser au cours de leur deuxième année. $S$ 'il arrive parfois que l'on trouve des sujets pesant tout au plus 20 grammes, par contre, d'autres, atteignent presque la livre : ce qui révèle avec quelle rapidité ils progressent Jorsqu'ils ont de la nourriture en abondiunce.

fin ce qui concerne le peuplement, au moyen de brochetons ayant moins d'une année d'existence, un seul et gros inconvénient existe : si l'étang ì empoissonner est à proximité de celui où les alevins ont été pêchés, tout sst parfait ; avec un peu de précaution, n’importe quel sujet, du plus petit au plus gros, peut être transporté sans crainte de mortalité, si la pèche a été faite irréprochablement. Par contre, s'il s'agit d'alevins ou filatons minuscules à déplacer à grande distance, parfois avec des moyens rudimentaires, il y a bien des chances pour qu'ils périssent tous en cours de route ou à la suite de leur immersion dans l'étang.

Pour pallier à cet inconvénient, il y a lieu, dans ce dernier cas, de choisir des brochetons de roo à 200 grammes, beaucoup plus résistants.

Dans certains cas, notamment lorsqu'intentionnellement, en raison de l'abondance des alevins en surnombre dans l'étang, on veut avoir unc pose importante, l'année suivante, il y a lien d'introduire des brochetons encore un peu plus gros.

Pour conclure, en régle générale, pour les Brochets : Il faut empoissonner toujours avec des brochetons les plus petits possible, à condition, toutefois, qu'ils ne risquent pas de périr au cours du transport, du fait de leur dólicatesse. 